\title{
Learning Shared Semantic Space for Speech-to-Text Translation
}

\author{
Chi Han ${ }^{1}$, Mingxuan Wang ${ }^{1}$, Heng $\mathrm{Ji}^{2}$, Lei Li ${ }^{1}$ \\ ${ }^{1}$ ByteDance AI Lab, ${ }^{2}$ University of Illinois at Urbana-Champaign \\ \{hanchi.me, wangmingxuan.89, lilei.02\}@bytedance.com \\ hengjieillinois.edu
}

\begin{abstract}
Having numerous potential applications and great impact, end-to-end speech translation (ST) has long been treated as an independent task, failing to fully draw strength from the rapid advances of its sibling - text machine translation (MT). With text and audio inputs represented differently, the modality gap has rendered MT data and its end-to-end models incompatible with their ST counterparts. In observation of this obstacle, we propose to bridge this representation gap with Chimera. By projecting audio and text features to a common semantic representation, Chimera unifies MT and ST tasks and boosts the performance on ST benchmarks, MuST-C and Augmented Librispeech, to a new state-of-the-art. Specifically, Chimera obtains 27.1 BLEU on MuST-C ENDE, improving the SOTA by a +1.9 BLEU margin. Further experimental analyses demonstrate that the shared semantic space indeed conveys common knowledge between these two tasks and thus paves a new way for augmenting training resources across modalities. 1
\end{abstract}

\section{Introduction}

Speech-to-text translation (ST) takes speech input in a source language and outputs text utterance in a target language. It has many real-world applications, including automatic video captioning, simultaneous translation for international conferences, etc. Traditional ST approaches cascade automatic speech recognition (ASR) and machine translation (MT) (Sperber et al., 2017, 2019; Zhang et al., 2019; Beck et al., 2019; Cheng et al., 2019). However, cascaded models often suffer from the issues of error propagation and translation latency. As a result, there have been a series of recent attempts on end-to-end speech-to-text translation (Liu et al.,

\footnotetext{
${ }^{1}$ All codes, data, and resources will be made released at https://github.com/Glaciohound/Chimera-SLT.
}

2019, 2018; Weiss et al., 2017; Bérard et al., 2018; Duong et al., 2016; Jia et al., 2019; Dong et al., 2021b; Wang et al., 2020b). The end-to-end approaches learn a single unified model, which is easier to deploy, has lower latency and could potentially reduce errors.

However, it remains a challenge for end-to-end ST to catch up with their cascaded counterparts in performance. We argue that the root cause is the gap between the two modalities, speech and text. Although they both encode human languages, they are dissimilar in both coding attributes (pitch, volume, and intonation versus words, affixes, and punctuation) and length (thousands of time frames versus tens of words). This issue is further coupled with the relatively smaller amount of parallel data for ST than for MT.

To tackle these challenges, we resort to making use of the additional available bilingual data for MT. Our hypothesis is, to better leverage MT data, an ideal model should be able to bridge the representations between speech and text. Motivated by this intuition, we propose Chimera, a text-speech shared semantic memory network. It learns a semantic memory by projecting features from both modalities into a shared semantic space. This approach unifies ST and MT workflows and thus has the advantage of leveraging massive MT corpora as a side boost in training. It can also use speechtext pairs to align the semantic memories from two modalities.

This idea of a unified text-speech representation also finds its neural basis as suggested by recent evidence from functional neuroimaging (van Atteveldt et al., 2004; Spitsyna et al., 2006; Shankweiler et al., 2008). Specifically, van Atteveldt et al. (2004); Spitsyna et al. (2006) identifies certain regions in brain that the processing stream for speech sounds and visual texts converge at. Shankweiler et al. (2008) further verifies that 
the size of such convergence sites correlates positively with the subjects' reading skills. Coincidentally, at these convergence sites also found regions responsive to downstream activities such as lexical and semantical word recognition (Binder et al., 2003) and spontaneous generation of speech (Blank et al., 2002). The evidence establishes the pivotal role of a modality-agnostic converged representation in language activities in brain.

This intuition lacks exploration in previous studies, with only a few exceptions (Indurthi et al., 2019; Liu et al., 2020), possibly due to the difficulties aforementioned and marginal improvements.

Our results show that Chimera achieves new state-of-the-art results on all of 8 translation directions in the benchmark datasets MuST-C and Augmented LibriSpeech. Specifically, Chimera obtains a 27.1 BLEU score on MuST-C EN-DE, which surpasses the best result ever reported by up to +1.9 BLEU. We also provide results under variations and ablations and validate our model design ideas by detailed analysis, as well as visualizing the semantic space Chimera has learned.

Our work makes the following contributions. First, we propose Chimera, which is able to bridge the modality gap between speech and text. Second, we derive a novel bi-modal contrastive training task to learn an alignment between semantic memories of two modalities. Finally, Chimera achieves a new state-of-the-art performance on the MuST-C benchmark and demonstrates its efficacy in learning modality-agnostic semantic representations.

\section{Related Work}

End-to-end ST Since its first proof-of-concept work (Bérard et al., 2016; Duong et al., 2016), solving Speech Translation in an end-to-end manner has attracted extensive attention (Vila et al., 2018; Salesky et al., 2018, 2019; Di Gangi et al., 2019b; Bahar et al., 2019a; Di Gangi et al., 2019c; Inaguma et al., 2020). Standard training techniques such as pretraining (Weiss et al., 2017; Bérard et al., 2018; Bansal et al., 2018; Stoian et al., 2020; Wang et al., 2020a; Pino et al., 2020), multi-task training (Vydana et al., 2021; Le et al., 2020; Tang et al., 2021), meta-learning (Indurthi et al., 2019), and curriculum learning (Kano et al., 2018; Wang et al., 2020b) have been applied. As ST data are expensive to collect, Jia et al. (2019); Pino et al. (2019); Bahar et al. (2019b) augment synthesized data from ASR and MT corpora. Methods utiliz- ing trained models, such as knowledge distillation (Liu et al., 2019) and model adaptation (Di Gangi et al., 2020), have also been shown to be effective. Among these attempts, (Indurthi et al., 2019; Le et al., 2020; Liu et al., 2020) are most related to ours, as they also attempt to train models on ASR or MT data. However, they both lack pivotal modules in model design to semantically bridge the gap between audio and text, and could thus suffer from modality mismatch in representations.

Cascaded ST The cascaded method is a more long-standing trend in ST (Sperber et al., 2017; Jan et al., 2018). To alleviate its innate problem of error propagation, Cheng et al. $(2018,2019)$ introduce synthetic ASR-related errors and perturbations. On the other hand, some post-processing techniques such as re-segmentation (Matusov et al., 2006), punctuation restoration (Fügen, 2008), and disfluency detection (Fitzgerald et al., 2009) are proposed to fix flaws or errors that occurred during the translation.

Cross-Lingual Techniques Techniques in multilingual tasks is also related to ours, as they aim at extracting common features out of sources from different representations (which, in this case, is language diversity) as well. However, multilingualism lacks key difficulties as observed in audio-text modality gap as discussed before. (Lu et al., 2018) and (Vazquez Carrillo et al., 2019) are early attempts by building an LSTM-based attentional interlingua. Yu et al. (2018); Yang et al. (2019) uses a similar cosine-based loss for multilingual training. Zhu et al. (2020) is probably more similar in method to ours, but Chimera is more simple in terms of model and objectives, and the memories in Chimera are additionally designed to focus on specific semantic categories.

\section{Proposed Method: Text-Speech Shared Semantic Memory Network}

\subsection{Speech Translation Overview}

An ST corpus usually consists of a set of triplet data $\mathcal{S}=\left\{\left(\mathbf{x}_{i}, \mathbf{z}_{i}, \mathbf{y}_{i}\right)\right\}$. Here $\mathbf{x}_{i}$ is the audio wave sequence, $\mathbf{z}_{i}$ is the transcript sequence and $\mathbf{y}_{i}$ is the translation sequence in the target language. As a benefit of shared semantic projection, Chimera is able to leverage large-scale MT training corpora $\mathcal{T}=\left\{\left(\mathbf{u}_{i}, \mathbf{v}_{i}\right)\right\}$, where $\mathbf{u}_{i}$ and $\mathbf{v}_{i}$ are the source and target text sequences. 


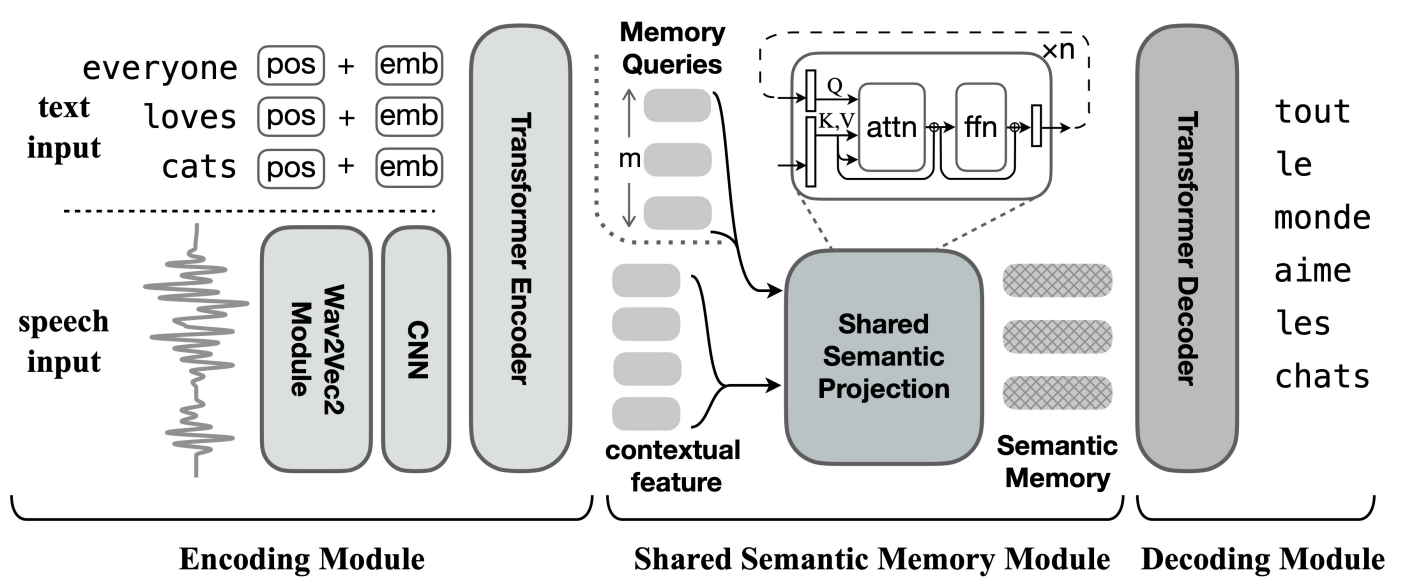

Figure 1: An overview of the proposed Chimera. The Encoder Module contains Word embedding for text input, and Wav2vec2 sub-module for speech input. The shared semantic projection Module uses its memory query to produce semantic memory with fixed-size representation from contextual features. The Decoder Module decodes translation from the semantic memory.

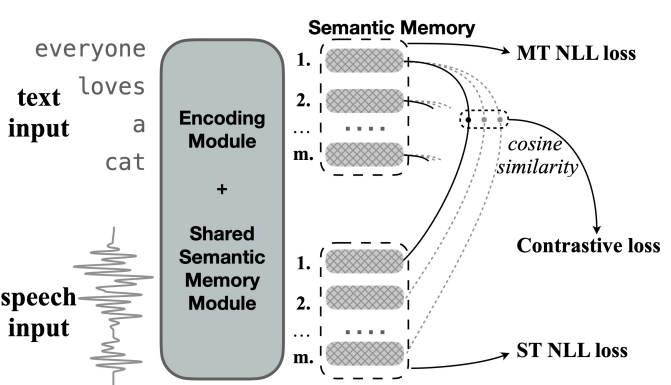

Figure 2: Besides MT and ST translation loss, we adopt a bi-modal contrastive loss to help aligning the semantic memories from text and speech. In short, among semantic memory elements from both sides of paired speech and transcript, the contrastive loss maximizes the cosine similarity between the same semantic memory element.

\subsection{Chimera Architecture}

Figure 1 illustrates the structure of Chimera. It consists of three major components, an encoding module, a shared semantic projection module, and a decoding module.

Encoding Module Different from that of a conventional ST model, the encoding module of Chimera accepts either speech or text as input. For text input, we use word embeddings plus positional embeddings. For speech input, we use a pretrained Wav2Vec2 (Baevski et al., 2020) to extract speech features. As the speech features can be very long, we apply an additional 1-dimensional strided CNN to reduce the length. Both speech and text branches share a common subsequent Transformer encoder (Vaswani et al., 2017). The final output of the en- coding module is contextual features.

Shared Semantic Projection Module Shared semantic projection plays a pivotal role in Chimera. The contextual features of speech and text may follow different distributions and of different lengths. Ideally, the shared semantic projection computes a constant number of semantic features as its output semantic memories.

This module take the contextual features out from the encoding module as input and then output semantic memories of fixed length $m$. It consists of $n$ attentional layers. It keeps a tuple of $m$ trainable input-dependent memory queries to indicate the types of desired semantic information, which is used as the initial "memories". Uni-modal contextual features serves as attention "keys" and "values", while memories serves as attention "queries". Memories are iteratively fed to the $n$ shared semantic projection layers, with each layer output used as input to next layer. The final output is used as the semantic memory.

$$
\begin{gathered}
\mathbf{I}_{0}=\mathbf{M}_{0} \in \mathbb{R}^{m \times d} \\
\mathbf{K}_{i}=\mathbf{V}_{i}=\hat{\mathbf{H}} \in \mathbb{R}^{l \times d} \\
\mathbf{I}_{i+1}=\mathbf{O}_{i}=\operatorname{Attn}\left(\mathbf{I}_{i}, \mathbf{K}_{i}, \mathbf{V}_{i}\right) \in \mathbb{R}^{m \times d}
\end{gathered}
$$

where $\mathbf{M}_{0}, \hat{\mathbf{H}}, \mathbf{I}_{i}$ and $\mathbf{O}_{i}$ denote the memory queries, contextual features, the input, and the output of each layer, respectively. $l$ is the length of contextual features. $d$ is the shared vector dimension. The top-most output $\mathbf{O}_{n}$ is finally fed into the decoding module. 
Decoding Module The decoding module contains a conventional Transformer decoder. The only difference is that the input is the fixed-size $\mathbf{O}_{n}$, which can possibly come from either speech or text.

\subsection{Training Objectives}

The training objective of Chimera consists of three aspects, with their supervision signals coming from speech-to-text translation data $\left\{\left(\mathbf{x}_{i}, \mathbf{y}_{i}\right)\right\}$, text machine translation data $\left(\left\{\mathbf{u}_{j}, \mathbf{v}_{j}\right)\right\}$ and $\left(\left\{\mathbf{z}_{i}, \mathbf{y}_{i}\right)\right\}$ and the speech-transcript pairs $\left\{\left(\mathbf{x}_{i}, \mathbf{z}_{i}\right)\right\}$.

Speech-to-Text Translation Training The workflow of Chimera in Speech Translation is straightforward. The training objective is negative log-likelihood on speech-to-text translation data $\left\{\left(\mathbf{x}_{i}, \mathbf{y}_{i}\right)\right\}$ as the loss function.

$$
\mathcal{L}_{\mathrm{ST}}=-\mathbb{E}_{\mathbf{x}, \mathbf{y}} \log \mathrm{P}(\mathbf{y} \mid \mathbf{x})
$$

Text Machine Translation Training Chimera is also able to train on MT corpus because of the unification of speech and text representations. Similar to ST training, the shared semantic projection module projects the contextual text features to the shared semantic space, which are then taken by the decoding module to output a translation.

$$
\mathcal{L}_{\mathrm{MT}}=-\mathbb{E}_{\mathbf{u}, \mathbf{v}} \log \mathrm{P}(\mathbf{v} \mid \mathbf{u})
$$

Bi-modal Contrastive Training The motivation of Chimera design is to bridge the speech and text representations. We introduce dual-modal contrastive training to learn an alignment between representations from speech and text as illustrated in Figure 2. First, semantic memories from two inputs are computed. Then for each text semantic memory, $\mathbf{M}_{i}^{t e x t}$, we compute its cosine similarities with all speech semantic memory $\left\{\cos \left(\mathbf{M}_{i}^{\text {text }}, \mathbf{M}_{j}^{\text {speech }}\right)\right\}$. They are then fed into a softmax function. The loss function maximizes the item from matched pairs $\left(\mathbf{M}_{i}^{\text {text }}, \mathbf{M}_{i}^{\text {speech }}\right)$. Finally, the loss is summed across all text memory items and vise versa.

$$
\begin{aligned}
\mathcal{L}_{\mathrm{ctr}}= & -\mathbb{E}_{\mathbf{x}, \mathbf{z}} \sum_{i} \ln \frac{e^{\tau \cos \left(\mathbf{M}_{i}^{\text {text }}, \mathbf{M}_{i}^{\text {speech }}\right)}}{\sum_{j} e^{\tau \cos \left(\mathbf{M}_{i}^{\text {text }}, \mathbf{M}_{j}^{\text {speech }}\right)}} \\
& -\mathbb{E}_{\mathbf{x}, \mathbf{z}} \sum_{j} \ln \frac{e^{\tau \cos \left(\mathbf{M}_{j}^{\text {speech }}, \mathbf{M}_{j}^{\text {text }}\right)}}{\sum_{i} e^{\tau \cos \left(\mathbf{M}_{j}^{\text {speech }}, \mathbf{M}_{i}^{\text {text }}\right)}}
\end{aligned}
$$

Intuitively, the contrastive loss forces the pair $\left(\mathbf{M}_{i}^{\text {text }}, \mathbf{M}_{i}^{\text {speech }}\right)$ to project semantic memories close to each other. In the meantime, the softmax function trains the model to maintain diversity among semantic memories.

The final loss is a weighted sum of each loss:

$$
\mathcal{L}=\lambda_{\mathrm{ST}} \mathcal{L}_{\mathrm{ST}}+\lambda_{\mathrm{MT}} \mathcal{L}_{\mathrm{MT}}+\lambda_{\text {ctr }} \mathcal{L}_{\text {ctr }}
$$

\section{Experiments}

We conduct experiments on the benchmark MuST$\mathrm{C}$ and, as a validation of model design, carry out ablation studies and visualize the representations Chimera has learned.

\subsection{Dataset and Preprocessing}

MuST-C (Di Gangi et al., 2019a) is a multilingual speech translation corpus with triplet data sources: source audio, transcripts, and text translations. MuST-C contains translations from English (EN) to 8 languages: Dutch (NL), French (FR), German (DE), Italian (IT), Portuguese (PT), Romanian (RO), Russian (RU), and Spanish (ES). With each pair consisting of at least 385 hours of audio recordings, to the best of our knowledge, MuST-C is currently the largest speech translation dataset available for each language pair. It includes data from English TED talks with manual transcripts and translations at the sentence level. We use the dev and tst-COMMON sets as our development and test data, respectively.

Augmented LibriSpeech Dataset (En-Fr) (Kocabiyikoglu et al., 2018) is composed of aligned e-books in French and their human reading in English. It provides typical triplet data of English speech, transcript and French text. Following the setting of (Liu et al., 2019), we utilize the $100 \mathrm{~h}$ hours of clean train set as training data, and use the original 2 hours of dev set and and 4 hours of test set.

Machine Translation Datasets After bridging the modality gap, Chimera has the potential power to utilize Machine Translation resources. Therefore we incorporate data from WMT, OpenSubtitles (Lison and Tiedemann, 2016) and OPUS100 (Zhang et al., 2020b) translation tasks. Specifically, we use WMT 2014 (Bojar et al., 2014) ${ }^{2}$ for EN-DE, EN-FR, EN-RU and EN-ES, WMT 2016 (Bojar et al., 2016) ${ }^{3}$ for EN-RO, and OPUS100 ${ }^{4}$ for

\footnotetext{
${ }^{2}$ downloadable at http://www.statmt.org/wmt14/translationtask.html

${ }^{3}$ downloadable at https://www.statmt.org/wmt16/translationtask.html

${ }^{4}$ downloadable at http://opus.nlpl.eu/opus-100.php
} 


\begin{tabular}{lccc|cccccccc}
\hline \multirow{2}{*}{ Model } & \multicolumn{9}{c}{ External Data } & \multicolumn{9}{c}{ MuST-C EN-X } \\
& Speech & ASR & MT & EN-DE & EN-FR & EN-RU & EN-ES & EN-IT & EN-RO & EN-PT & EN-NL \\
\hline FairSeq ST $^{\dagger}$ & $\times$ & $\times$ & $\times$ & 22.7 & 32.9 & 15.3 & 27.2 & 22.7 & 21.9 & 28.1 & 27.3 \\
Espnet ST $^{\ddagger}$ & $\times$ & $\times$ & $\times$ & 22.9 & 32.8 & 15.8 & 28.0 & 23.8 & 21.9 & 28.0 & 27.4 \\
AFS $^{\star}$ & $\times$ & $\times$ & $\times$ & 22.4 & 31.6 & 14.7 & 26.9 & 23.0 & 21.0 & 26.3 & 24.9 \\
Dual-Decoder $^{\diamond}$ & $\times$ & $\times$ & $\times$ & 23.6 & 33.5 & 15.2 & 28.1 & 24.2 & 22.9 & $\mathbf{3 0 . 0}$ & 27.6 \\
STATST $^{\sharp}$ & $\times$ & $\times$ & $\times$ & 23.1 & - & - & - & - & - & - & - \\
MAML $^{b}$ & $\times$ & $\times$ & $\checkmark$ & 22.1 & 34.1 & - & - & - & - & - & - \\
Self-Training $^{\circ}$ & $\checkmark$ & $\checkmark$ & $\times$ & 25.2 & 34.5 & - & - & - & - & - & - \\
W2V2-Transformer $^{*}$ & $\checkmark$ & $\times$ & $\times$ & 22.3 & 34.3 & 15.8 & 28.7 & 24.2 & 22.4 & 29.3 & 28.2 \\
\hline Chimera Mem-16 $_{\text {Chimera }}$ & $\checkmark$ & $\times$ & $\checkmark$ & 25.6 & 35.0 & 16.7 & 30.2 & 24.0 & 23.2 & 29.7 & 28.5 \\
& $\checkmark$ & $\times$ & $\checkmark$ & $\mathbf{2 7 . 1}$ & $\mathbf{3 5 . 6}$ & $\mathbf{1 7 . 4}$ & $\mathbf{3 0 . 6}$ & $\mathbf{2 5 . 0}$ & $\mathbf{2 4 . 0}$ & $\mathbf{3 0 . 2}$ & $\mathbf{2 9 . 2}$ \\
\hline
\end{tabular}

Table 1: Main results on tst-COMMON subset on all 8 languages in MuST-C dataset. "Speech" denotes unlabeled audio data. $\bullet:$ the result uses a mixed WMT14+OpenSubtitles data for MT pre-training. EN-DE Among the baselines, ${ }^{\dagger}$ shows results from Ott et al. (2019), ${ }^{\ddagger}$ from Inaguma et al. (2020), ${ }^{\star}$ from Zhang et al. (2020a), $\diamond$ from Le et al. (2020), ${ }^{\sharp}$ from Liu et al. (2020), ${ }^{b}$ from Indurthi et al. (2019), and ${ }^{\circ}$ from Pino et al. (2019). ${ }^{*}$ shows results of a simple baseline model by combining a Wav2Vec2 module (Baevski et al., 2020) and a Transformer model, which could be viewed as the "no external data" version of Chimera.

\begin{tabular}{cccc|c}
\hline \multirow{2}{*}{ Model } & \multicolumn{4}{c}{ External Data } \\
& Speech & ASR & MT & EN-FR \\
\hline W2V2-T $^{*}$ & $\checkmark$ & $\times$ & $\times$ & 6.4 \\
TCEN $^{\dagger}$ & $\times$ & $\times$ & $\times$ & 17.1 \\
LSTM $^{\ddagger}$ & $\times$ & $\checkmark$ & $\checkmark$ & 17.0 \\
AFS $^{\circ}$ & $\times$ & $\times$ & $\times$ & 17.2 \\
Multilingual $^{\star}$ & $\times$ & $\checkmark$ & $\times$ & 17.6 \\
Transformer $^{\perp}$ & $\times$ & $\checkmark$ & $\times$ & 17.7 \\
Curiculum $^{\perp}$ & $\times$ & $\checkmark$ & $\times$ & 18.0 \\
COSTT $^{b}$ & $\times$ & $\times$ & $\checkmark$ & 18.2 \\
LUT $^{\diamond}$ & $\times$ & $\checkmark$ & $\times$ & 18.3 \\
STAST $^{\sharp}$ & $\times$ & $\checkmark$ & $\times$ & 18.7 \\
\hline Chimera $^{+}$ & $\checkmark$ & $\times$ & $\checkmark$ & $\mathbf{1 9 . 4}$ \\
\hline
\end{tabular}

We additionally evaluate OpenSubtitles as EN-DE MT data to test the impact of MT corpus selection. WMT 2014 dataset provides at least 4 million sentences of translation data in each language pair. WMT 2016 contains less, around 600k for EN-RO direction. OPUS100 has around $1 \mathrm{M}$ sentences for each sentence pair. OpenSubtitles provides 22M sentences for EN-DE.

Pre-processing of Data and Evaluation For speech input, the 16-bit raw wave sequences are normalized by a factor of $2^{15}$ to the range of $[-1,1)$.

For text input, on each translation pair, all texts (ST transcripts and translation, and MT source and target texts) are pre-processed in the same way.

Table 2: Results on LibriSpeech English-French dataset. ${ }^{*}$ is the same W2V2-Transformer baseline as in Table 1. ${ }^{\dagger}$ is from Wang et al. (2020a), ${ }^{\ddagger}$ from Bahar et al. (2019b), * from Inaguma et al. (2019), two baselines under ${ }^{\perp}$ from Wang et al. (2020b), ${ }^{b}$ from Dong et al. (2021a), $\diamond$ from Dong et al. (2021b), ${ }^{\circ}$ from Zhang et al. (2020a) and ${ }^{\sharp}$ from Liu et al. (2020).

\begin{tabular}{lccc|c}
\hline \multirow{2}{*}{ Model } & \multicolumn{3}{c}{ External Data } & MuST-C \\
& Speech & ASR & MT & EN-DE \\
\hline W2V2-T + Dec PT & $\checkmark$ & $\times$ & WMT14 & 22.2 \\
W2V2-T + KD & $\checkmark$ & $\times$ & WMT14 & 24.6 \\
Chimera & $\checkmark$ & $\times$ & WMT14 & $\mathbf{2 6 . 3}$ \\
\hline
\end{tabular}

Table 3: Comparison with other baselines utilizing external MT data on MuST-C EN-DE. "Dec PT" pretrains decoder on MT corpus; "KD" adopts the knowledge distillation technique used in Liu et al. (2019)

EN-PT, EN-IT, and EN-NL, as pretraining corpora. from words, and normalized. Non-print punctuation is removed. The sentences are then tokenized with Moses tokenizer ${ }^{5}$. We filter out samples whose number of source or target tokens is over 250 and whose ratio of source and target text lengths is outside range $[2 / 3,3 / 2]$. For sub-wording, we use a unigram sentencepiece ${ }^{6}$ model with a dictionary size of 10000 . On each translation direction, The sentencepiece model is learned on all text data from both ST and MT corpora. The dictionary is shared across MT and ST and across source and target languages.

The performance is evaluated with BLEU (Papineni et al., 2002) using sacreBLEU ${ }^{7}$. We average

\footnotetext{
${ }^{5}$ https://github.com/moses-

smt/mosesdecoder/blob/master/scripts/tokenizer/tokenizer.perl

${ }^{6} \mathrm{https}: / /$ github.com/google/sentencepiece

${ }^{7}$ https://github.com/mjpost/sacrebleu, with configuration
} 


\begin{tabular}{cc|cc}
\hline S.S. Projection & Decoder & EN-DE & EN-FR \\
\hline- & - & 25.6 & 35.0 \\
Fixed & - & 24.3 & 34.3 \\
- & Fixed & 24.2 & 33.4 \\
Fixed & Fixed & 23.8 & 33.1 \\
\hline
\end{tabular}

Table 4: Performance of Mem-16 Chimera when freezing different modules in fine-tuning. S.S. Projection is abbreviation for shared semantic projection. "Fixed" indicates that weights in this module are not updated during fine-tuning, and "-" means otherwise. The results demonstrate that freezing modules indeed hampers the model's ability to adapt, but the weights pretrained on MT are already highly informative for ST.

7 consecutive checkpoints around the one of the best dev loss and adopt a beam size of 10 .

\subsection{Model Configuration}

For text input, we use 512-dimensional word embeddings plus sinusoidal positional embeddings. For audio input, the Wav2Vec2 Module follows the base configuration in Baevski et al. (2020). It uses parameters pretrained on LibriSpeech audio data only. The 1-dimensional CNN for speech features has 2 layers with stride size 2 , kernel size 5 , padding 2, and hidden dimension 1024 .

The shared Transformer encoder consists of 6 layers. The memory queries are 64 512dimensional vectors. The parameters of shared semantic projection resemble a 3-layer Transformer encoder. The Transformer decoder has 6 layers. Each of these Transformer layers, except for those in the Wav2Vec2 module, has an embedding dimension of 512, a hidden dimension of 512, and 8 attention heads.

In both pretraining and fine-tuning stages, we use an Adam optimizer with $\beta_{1}=0.9, \beta_{2}=0.98$, and $4 \mathrm{k}$ warm-up updates. We apply an inverse square root schedule algorithm for the learning rate. In MT pretraining, the learning rate is $5 \mathrm{e}-4$, the maximum number of updates is $300 \mathrm{k}$, with at most 33k input tokens per batch. In ST pretraining, the learning rate is $1 \mathrm{e}-4$, the maximum number of updates is $150 \mathrm{k}$, with at most $16 \mathrm{M}$ source audio frames per batch. The loss weights $\lambda_{\mathrm{ST}}, \lambda_{\mathrm{MT}}$ and $\lambda_{\text {ctr }}$ are all set to 1 .

We also show results on a base version of Chimera, for which the memory queries are only 16 512-dimensional vectors (codenamed "Mem-16").

of 13a tokenzier, case-sensitiveness and full punctuation

\begin{tabular}{cc|cc}
\hline MT & Contrastive & EN-DE & EN-FR \\
\hline$\checkmark$ & $\checkmark$ & 25.6 & 35.0 \\
$\checkmark$ & $\times$ & 25.0 & 34.6 \\
$\times$ & $\checkmark$ & 24.7 & 34.6 \\
$\times$ & $\times$ & 25.1 & 34.6 \\
\hline
\end{tabular}

Table 5: BLEU scores of Mem-16 Chimera on MuST$\mathrm{C}$ tst-COMMON set without one or both of auxiliary tasks. " $\times$ " means this task is not used during finetuning, and " $\checkmark$ " means othersize. "Contrastive" is the bi-modal contrastive task. The removal of one or both of tasks greatly harms the model's performance on both language pairs.

Because of the training efficiency and simplicity, all ablation studies and visualizations adopted the Mem-16 configuration if not stated otherwise.

Both Chimera and Chimera Mem-16 contain around $165 \mathrm{M}$ parameters. The whole training process for one trial on 8 Nvidia Tesla-V100 GPUs generally takes $20-40$ hours according to the translation direction.

\subsection{Benchmark Experiments}

Training We train Chimera in a pretrain - fine-tune manner. In the first stage, we pretrain Chimera on MT datasets so as to leverage additional sources of training data, as well as provide a better initialization point. In the fine-tuning stage, we adopt multi-task training as described in Section3.3. In addition to the conventional ST task, Chimera is also fine-tuned on MT and bi-modal contrastive task to align inputs from speech and text.

Baselines We include as baselines the speech transformer model from (Ott et al., 2019), Espnet result from (Inaguma et al., 2020), adaptive feature selection method from (Zhang et al., 2020a), dualdecoder Transformer from (Le et al., 2020) and Modality-Agnostic Meta-Learning from (Indurthi et al., 2019) in Table 1. We also provide a series of baseline results of a simple combination of Wav2Vec2 (Baevski et al., 2020) and Transformer. It could be viewed Chimera without external MT pre-training, with still competitive but not SOTA results.

To verify the effectiveness of our training technique, we also compare with other baselines able to leverage external MT corpus in Table 3.

Results The experiment results are shown in Table 1 and 2. Our Chimera achieves state-of-the-art performance on all language pairs, even though we do 


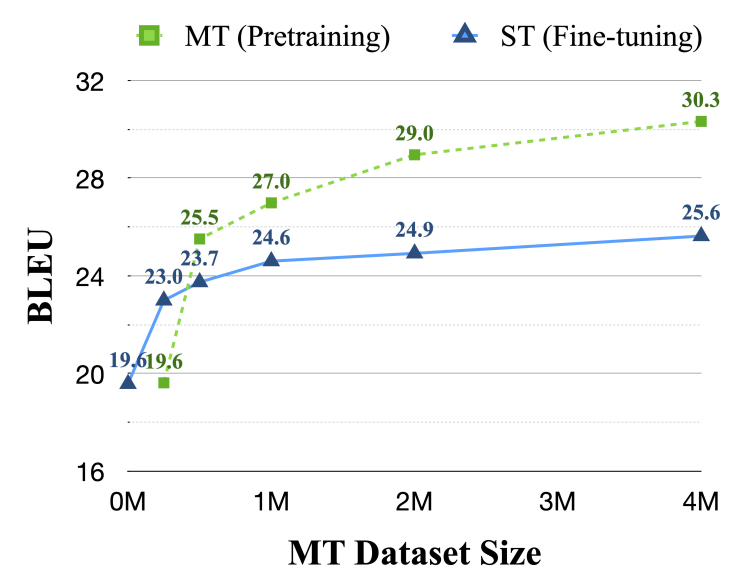

(a) Chimera Mem-16 on WMT14

\begin{tabular}{cc|cc}
\hline Corpora & Size & MT & ST \\
\hline WMT14 & $4 \mathrm{M}$ & 32.1 & 26.3 \\
WMT14 + OpenSubtitles & $26 \mathrm{M}$ & 32.9 & 27.1 \\
\hline
\end{tabular}

(b) Chimera w/ and w/o OpenSubtitles

Figure 3: Curve of MuST-C EN-DE tst-COMMON BLEU scores on Chimera against the amount of MT data used during pretraining. (a) shows Chimera Mem16's performance on WMT14. Blue triangles are the speech translation BLEU scores, and green squares are transcript-translation BLEU scores after MT pretraining. (b) shows how Chimera $(M=64)$ behaves with or without OpenSubtitles data.

not utilize Google Translate results on Augmented Librispeech as most baselines. EN-DE results of Chimera uses WMT14+OpenSubtitles for MT pretrainng, while a detailed ablation study on the effect of MT data can be found in Section 4.4. Note that the improvement on EN-PT is not so significant as EN-DE and EN-FR. We attribute this to the data discrepancy between OPUS100 and MuST-C. A large number of sentences in OPUS100 are from movie subtitles, which are more informal, contain repeated sentences, and cover different topics from those in MuST-C public speeches.

In Table 3, under the same data condition, Chimera outperforms other techniques such as decoder pre-training and knowledge distillation (Liu et al., 2019).

\subsection{Ablation Studies and Visualizations}

Knowledge Shared across Tasks One potential benefit in our design is that the shared semantic space can hold common knowledge shared across ST and MT tasks. To validate this motivating idea, we analyze the model's behavior while manipu-

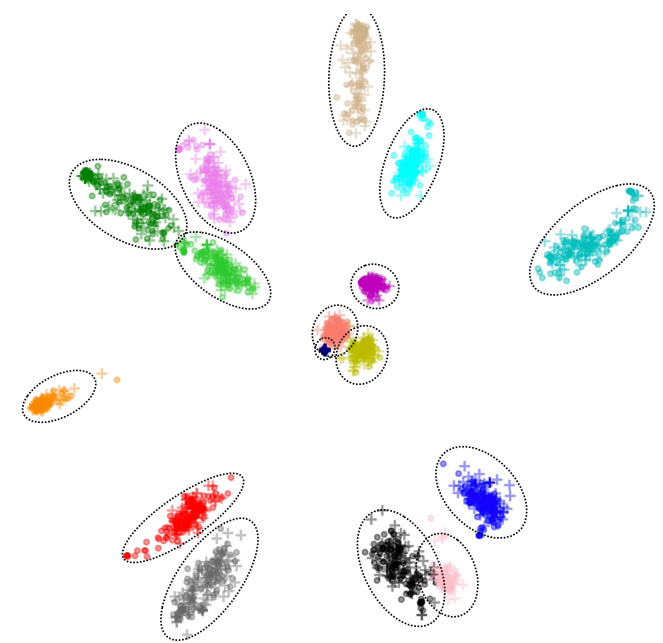

Figure 4: 2-dimensional PCA projection of the semantic memories in Mem16 Chimera across different samples. Each colored cluster (circled out) represents a semantic memory element,. A “." corresponds to a speech semantic memory, and a "+" marks a text one.

lating its modules. If certain weights pretrained during the MT task also contain meaningful information for ST, fixing them should not greatly harm the model's performance.

Specifically, after MT pretraining, we fix certain modules and do not update their weights during fine-tuning. We choose to fix the weights in the shared semantic projection module, the decoding module, or both of them.

Table 4 shows the results. After freezing modules, the results on both EN-DE and EN-FR drop slightly. This demonstrates that freezing weights indeed hampers the model's ability to adapt from MT to ST dataset. But the decreased scores are still comparable to many of the best results in Table 1 . This validates the effectiveness of shared semantic space, and indicates that the weights pretrained on MT are already informative enough for Chimera to still generalize sufficiently well on ST task.

Multi-task Training One advantage of bridging the modality gap is that the model can fully benefit from training on auxiliary tasks with more data, such as those mentioned in Section 3.2. To evaluate their impacts, we conduct another ablation study on EN-DE and EN-FR.. Either or both of the auxiliary tasks are not used during fine-tuning.

The results of this ablation are presented in Table 5. Here we can see a significant decrease (with, for example, $p=0.020$ in one-tailed Student's t-test comparing row 1 and 2) in BLEU scores when either of the auxiliary tasks is abandoned. Although 


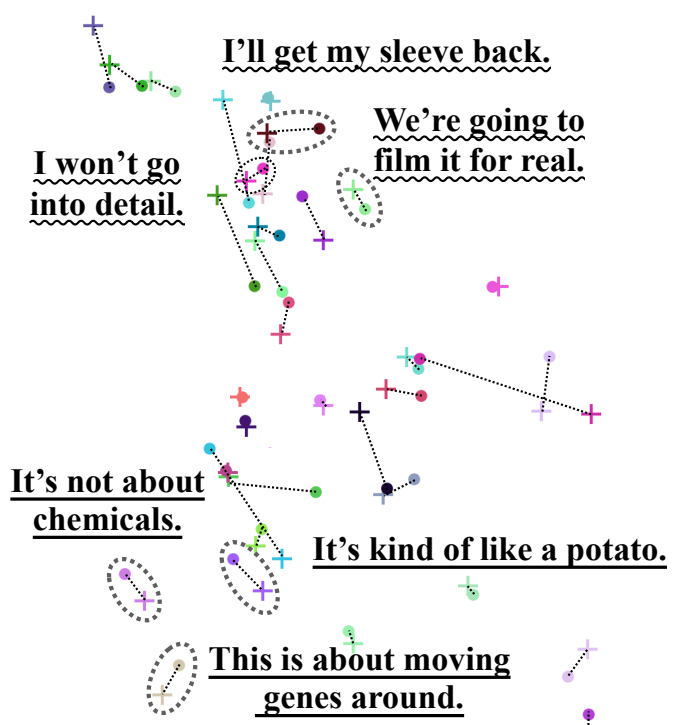

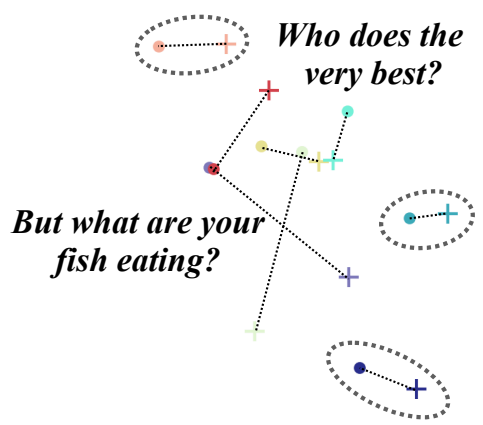

So what do we have to do?

Figure 5: A visualization of one particular semantic memory in Mem-16 Chimera no different samples and modalities. "." marks speech representations, and "+" marks text representations. Marks of the same color come from the same speech-transcript pair and are linked with dashed lines. Some of speech-transcript pairs are circled together and annotated with their transcripts. Three fonts distinguish three groups of transcripts of similar patterns.

the bi-model contrastive loss is not directly related to the ST task, a poor alignment between semantic memories of speech and text hinders Chimera from leveraging the shared knowledge across two tasks. When the MT task is removed, the drop in BLEU scores is also huge. This could be explained that during fine-tuning, the auxiliary MT task is necessary for keeping shared knowledge from being forgotten.

It is interesting to observe that abandoning both tasks produces results similar to abandoning either one of the tasks. This suggests that the two auxiliary tasks only have effects when combined with each other: only when both using MT task to maintain the pretrained parameters from forgetting, as well as using the bi-modal contrastive task to align between speech and text representations, can Chimera benefit from shared knowledge in MT pretraining.

Additional Machine Translation Data We attribute the gain in the performance of Chimera mainly to the pretraining on MT data. One evidence is the performance gain when using the larger OpenSubtitles as MT corpus for EN-DE in 1. To further probe the influence of the additional MT data, we vary the amount of MT data available during pretraining on EN-DE direction. The results are plotted in Figure 3.

As the size of additional MT dataset increases, the MuST-C BLEU score improves significantly.
This confirms the importance of massive highquality MT data for pretraining Chimera. The results also help explain the relatively inferior scores on EN-PT in Table 1 which uses the OPUS100 dataset in pretraining.

Visualization of Semantic Memories The shared semantic projection is designed to only extract semantic categories of information necessary for decoding, regardless of the input modality. In this way, it can bridge the different representations of speech and text during computation and facilitate knowledge sharing between MT and ST.

To validate this motivation, we visualize them with Principal Component Analysis (PCA) in Figure 4. Up to 100 speech-transcript paired samples are randomly chosen from the valid set. We record vector values of 16 semantic memories from Chimera Mem-16 when inputs are speeches or transcripts, and apply 2-dimensional PCA. The 16 semantic memories are distinguished by 16 colors. Every "." corresponds to a semantic memory from speech, and each "+" is a semantic memory from the text. It is clear that the semantic memories are highly clustered, everyone of which learns a particular region. Speech and text representations are also projected close within the same region, proving the model's ability to ignore representation differences and bridge the modality gap.

To take a closer view of the structure of each semantic memory subspace, we randomly choose one 


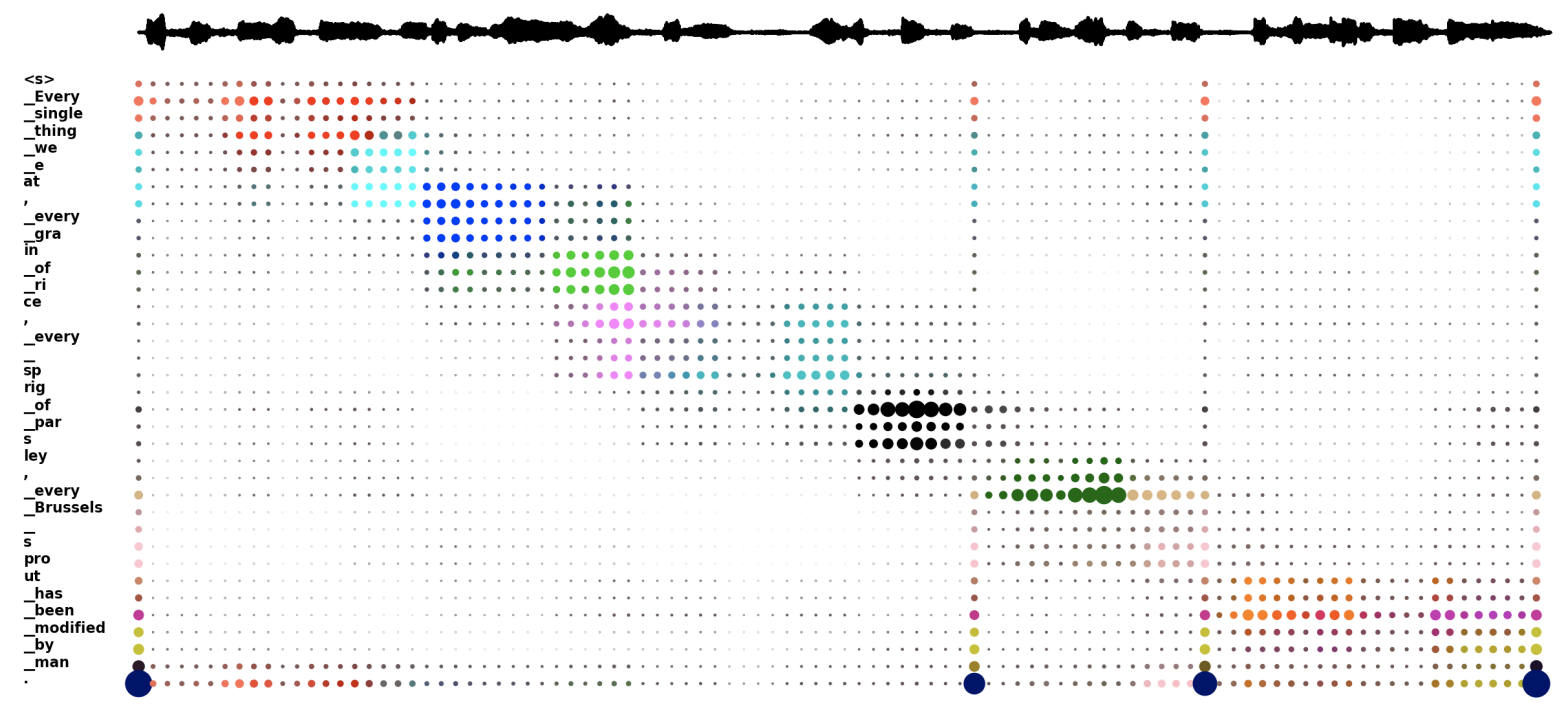

Figure 6: Visualization of the final-layer attention of $M=16$ memories on inputs, and their alignment between each other. A pair of audio and its transcript is fed to Chimera Mem-16. The area of each dot is linear to dot product between two attention vectors. The color is a linear interpolation of $M$ indicative colors as in Fig 4, with mixing weights linear to the Hadamard product of attention vectors. (Best viewed in color)

semantic memory and apply PCA to its corresponding cluster. The results are visualized in Figure 5. These samples come from 50 speech-transcript pairs. Each pair of speech (“".) and transcript ("+") share the same color and are linked through dashed lines.

Two interesting properties could be observed. First, paired speech and transcript inputs are again close to each other, even though they are coming from different modalities. Second, the visualized representations are organized according to their semantic or syntactic patterns. We recognize several clusters in the figure, and annotate their transcripts with different fonts. The three annotations at the upper-right corner (Italic font) are all questions; those at the upper-left corner (wavy underlined font) all follow a simple future tense; at the bottomleft corner of the figure (underlined font) is another cluster of sentences of copular verbs. This proves that the shared semantic space that Chimera has learned is well-structured, and thus validates our model design.

\section{Visualization of Inter-Modal Attention Align-} ment "Attention" is the internal mechanism of Transformer based modules. In the design of Chimera, attention is used for extracting $M$ key semantic categories of features from input. To investigate whether these extracted features is indeed semantic, we further visualized the similarity between attention on paired audio and text in Fig 6.
Here the colors, which distinguish different memories attending to inputs, is clustered on sequence and distributed close to the diagonal, demonstrating an alignment between matching tokens in two modalities. Here we also observe four beaming columns, where the full stop mark in text aligns with pauses in audio. This is an indication of semantic rather than positional essence of the memories.

\section{Conclusions and Future Work}

In this paper, we propose Chimera, a model capable of learning a text-speech shared semantic memory network for bridging the gap between speech and text representations. Being able to leverage a large amount of external Machine Translation data, Chimera achieves new state-of-the-art performance on the MuST-C dataset on all 8 languages. Additional experiment results also demonstrate its ability to learn a well-structured shared semantic space as well as effectively share learned knowledge across MT and ST, and validate our design of auxiliary tasks.

In the future, we will focus on deriving a better task to tightly align speech and text representations. Also, the workflows of MT and ST are only partially shared in Chimera, which still requires the model to adapt to ST when switching to the finetuning stage. So it remains a challenge to better couple their computation graphs in future designs. 


\section{References}

Alexei Baevski, Henry Zhou, Abdelrahman Mohamed, and Michael Auli. 2020. wav2vec 2.0: A framework for self-supervised learning of speech representations.

Parnia Bahar, Tobias Bieschke, and Hermann Ney. 2019a. A comparative study on end-to-end speech to text translation. In 2019 IEEE Automatic Speech Recognition and Understanding Workshop (ASRU), pages 792-799. IEEE.

Parnia Bahar, Albert Zeyer, Ralf Schlüter, and Hermann Ney. 2019b. On using specaugment for end-to-end speech translation. arXiv preprint arXiv:1911.08876.

Sameer Bansal, Herman Kamper, Karen Livescu, Adam Lopez, and Sharon Goldwater. 2018. Pretraining on high-resource speech recognition improves low-resource speech-to-text translation. arXiv preprint arXiv:1809.01431.

Daniel Beck, Trevor Cohn, and Gholamreza Haffari. 2019. Neural speech translation using lattice transformations and graph networks. In Proceedings of the Thirteenth Workshop on Graph-Based Methods for Natural Language Processing (TextGraphs-13), pages 26-31.

Alexandre Bérard, Laurent Besacier, Ali Can Kocabiyikoglu, and Olivier Pietquin. 2018. End-toend automatic speech translation of audiobooks. In ICASSP, pages 6224-6228. IEEE.

Alexandre Bérard, Olivier Pietquin, Christophe Servan, and Laurent Besacier. 2016. Listen and translate: A proof of concept for end-to-end speech-to-text translation. arXiv preprint arXiv:1612.01744.

Jeffrey R Binder, Kristen A McKiernan, Melanie E Parsons, Chris F Westbury, Edward T Possing, Jacqueline N Kaufman, and Lori Buchanan. 2003. Neural correlates of lexical access during visual word recognition. Journal of cognitive neuroscience, 15(3):372-393.

S Catrin Blank, Sophie K Scott, Kevin Murphy, Elizabeth Warburton, and Richard JS Wise. 2002. Speech production: Wernicke, broca and beyond. Brain, 125(8):1829-1838.

Ondřej Bojar, Christian Buck, Christian Federmann, Barry Haddow, Philipp Koehn, Johannes Leveling, Christof Monz, Pavel Pecina, Matt Post, Herve Saint-Amand, et al. 2014. Findings of the 2014 workshop on statistical machine translation. In Proceedings of the ninth workshop on SMT, pages 1258.

Ondřej Bojar, Rajen Chatterjee, Christian Federmann, Yvette Graham, Barry Haddow, Matthias Huck, Antonio Jimeno Yepes, Philipp Koehn, Varvara Logacheva, Christof Monz, et al. 2016. Findings of the 2016 conference on machine translation. In Proceedings of the First Conference on Machine Translation, pages 131-198.

Qiao Cheng, Meiyuan Fang, Yaqian Han, Jin Huang, and Yitao Duan. 2019. Breaking the data barrier: Towards robust speech translation via adversarial stability training. arXiv preprint arXiv:1909.11430.

Yong Cheng, Zhaopeng Tu, Fandong Meng, Junjie Zhai, and Yang Liu. 2018. Towards robust neural machine translation. arXiv preprint arXiv:1805.06130.

Mattia A Di Gangi, Roldano Cattoni, Luisa Bentivogli, Matteo Negri, and Marco Turchi. 2019a. Must-c: a multilingual speech translation corpus. In 2019 Conference of the North American Chapter of the Association for Computational Linguistics: Human Language Technologies, pages 2012-2017. Association for Computational Linguistics.

Mattia A Di Gangi, Matteo Negri, and Marco Turchi. 2019b. Adapting transformer to end-to-end spoken language translation. In INTERSPEECH 2019, pages 1133-1137. International Speech Communication Association (ISCA).

Mattia A Di Gangi, Viet-Nhat Nguyen, Matteo Negri, and Marco Turchi. 2020. Instance-based model adaptation for direct speech translation. In ICASSP 2020-2020 IEEE International Conference on Acoustics, Speech and Signal Processing (ICASSP), pages 7914-7918. IEEE.

Mattia Antonino Di Gangi, Matteo Negri, Roldano Cattoni, Dessi Roberto, and Marco Turchi. 2019c. Enhancing transformer for end-to-end speech-to-text translation. In Machine Translation Summit XVII, pages 21-31. European Association for Machine Translation.

Qianqian Dong, Mingxuan Wang, Hao Zhou, Shuang $\mathrm{Xu}, \mathrm{Bo} \mathrm{Xu}$, and Lei Li. 2021a. Consecutive decoding for speech-to-text translation. In The Thirty-fifth AAAI Conference on Artificial Intelligence, AAAI.

Qianqian Dong, Rong Ye, Mingxuan Wang, Hao Zhou, Shuang Xu, Bo Xu, and Lei Li. 2021b. "listen, understand and translate": Triple supervision decouples end-to-end speech-to-text translation. In Proceedings of the AAAI Conference on Artificial Intelligence, volume 35, pages 12749-12759.

Long Duong, Antonios Anastasopoulos, David Chiang, Steven Bird, and Trevor Cohn. 2016. An attentional model for speech translation without transcription. In NAACL, pages 949-959.

Erin Fitzgerald, Keith B Hall, and Frederick Jelinek. 2009. Reconstructing false start errors in spontaneous speech text.

Christian Fügen. 2008. A system for simultaneous translation of lectures and speeches. Ph.D. thesis, Verlag nicht ermittelbar. 
Hirofumi Inaguma, Kevin Duh, Tatsuya Kawahara, and Shinji Watanabe. 2019. Multilingual end-to-end speech translation. In 2019 IEEE Automatic Speech Recognition and Understanding Workshop (ASRU), pages 570-577. IEEE.

Hirofumi Inaguma, Shun Kiyono, Kevin Duh, Shigeki Karita, Nelson Enrique Yalta Soplin, Tomoki Hayashi, and Shinji Watanabe. 2020. Espnet-st: Allin-one speech translation toolkit. arXiv preprint arXiv:2004.10234.

Sathish Indurthi, Houjeung Han, Nikhil Kumar Lakumarapu, Beomseok Lee, Insoo Chung, Sangha Kim, and Chanwoo Kim. 2019. Data efficient direct speech-to-text translation with modality agnostic meta-learning. arXiv preprint arXiv:1911.04283.

Niehues Jan, Roldano Cattoni, Stüker Sebastian, Mauro Cettolo, Marco Turchi, and Marcello Federico. 2018. The iwslt 2018 evaluation campaign. In International Workshop on Spoken Language Translation, pages 2-6.

Ye Jia, Melvin Johnson, Wolfgang Macherey, Ron J Weiss, Yuan Cao, Chung-Cheng Chiu, Naveen Ari, Stella Laurenzo, and Yonghui Wu. 2019. Leveraging weakly supervised data to improve end-to-end speech-to-text translation. In ICASSP, pages 7180 7184. IEEE.

Takatomo Kano, Sakriani Sakti, and Satoshi Nakamura. 2018. Structured-based curriculum learning for endto-end english-japanese speech translation. arXiv preprint arXiv:1802.06003.

Ali Can Kocabiyikoglu, Laurent Besacier, and Olivier Kraif. 2018. Augmenting librispeech with french translations: A multimodal corpus for direct speech translation evaluation. arXiv preprint arXiv:1802.03142.

Hang Le, Juan Pino, Changhan Wang, Jiatao Gu, Didier Schwab, and Laurent Besacier. 2020. Dualdecoder transformer for joint automatic speech recognition and multilingual speech translation. arXiv preprint arXiv:2011.00747.

Pierre Lison and Jörg Tiedemann. 2016. Opensubtitles2016: Extracting large parallel corpora from movie and tv subtitles.

Dan Liu, Junhua Liu, Wu Guo, Shifu Xiong, Zhiqiang Ma, Rui Song, Chongliang Wu, and Quan Liu. 2018. The ustc-nel speech translation system at iwslt 2018. arXiv preprint arXiv:1812.02455.

Yuchen Liu, Hao Xiong, Zhongjun He, Jiajun Zhang, Hua Wu, Haifeng Wang, and Chengqing Zong. 2019. End-to-end speech translation with knowledge distillation. arXiv preprint arXiv:1904.08075.

Yuchen Liu, Junnan Zhu, Jiajun Zhang, and Chengqing Zong. 2020. Bridging the modality gap for speech-to-text translation. arXiv preprint arXiv:2010.14920.
Yichao Lu, Phillip Keung, Faisal Ladhak, Vikas Bhardwaj, Shaonan Zhang, and Jason Sun. 2018. A neural interlingua for multilingual machine translation. arXiv preprint arXiv:1804.08198.

Evgeny Matusov, Arne Mauser, and Hermann Ney. 2006. Automatic sentence segmentation and punctuation prediction for spoken language translation. In International Workshop on Spoken Language Translation (IWSLT) 2006.

Myle Ott, Sergey Edunov, Alexei Baevski, Angela Fan, Sam Gross, Nathan Ng, David Grangier, and Michael Auli. 2019. fairseq: A fast, extensible toolkit for sequence modeling. In Proceedings of NAACL-HLT 2019: Demonstrations.

Kishore Papineni, Salim Roukos, Todd Ward, and WeiJing Zhu. 2002. Bleu: a method for automatic evaluation of machine translation. In Proceedings of the 40th ACL, pages 311-318.

Juan Pino, Liezl Puzon, Jiatao Gu, Xutai Ma, Arya D McCarthy, and Deepak Gopinath. 2019. Harnessing indirect training data for end-to-end automatic speech translation: Tricks of the trade. arXiv preprint arXiv:1909.06515.

Juan Pino, Qiantong Xu, Xutai Ma, Mohammad Javad Dousti, and Yun Tang. 2020. Self-training for end-to-end speech translation. arXiv preprint arXiv:2006.02490.

Elizabeth Salesky, Susanne Burger, Jan Niehues, and Alex Waibel. 2018. Towards fluent translations from disfluent speech. In 2018 (SLT), pages 921-926. IEEE.

Elizabeth Salesky, Matthias Sperber, and Alex Waibel. 2019. Fluent translations from disfluent speech in end-to-end speech translation. arXiv preprint arXiv:1906.00556.

Donald Shankweiler, W Einar Mencl, David Braze, Whitney Tabor, Kenneth R Pugh, and Robert K Fulbright. 2008. Reading differences and brain: Cortical integration of speech and print in sentence processing varies with reader skill. Developmental neuropsychology, 33(6):745-775.

Matthias Sperber, Graham Neubig, Jan Niehues, and Alex Waibel. 2017. Neural lattice-to-sequence models for uncertain inputs. arXiv preprint arXiv:1704.00559.

Matthias Sperber, Graham Neubig, Ngoc-Quan Pham, and Alex Waibel. 2019. Self-attentional models for lattice inputs. arXiv preprint arXiv:1906.01617.

Galina Spitsyna, Jane E Warren, Sophie K Scott, Federico E Turkheimer, and Richard JS Wise. 2006. Converging language streams in the human temporal lobe. Journal of Neuroscience, 26(28):7328-7336. 
Mihaela C Stoian, Sameer Bansal, and Sharon Goldwater. 2020. Analyzing asr pretraining for low-resource speech-to-text translation. In ICASSP 2020-2020 IEEE International Conference on Acoustics, Speech and Signal Processing (ICASSP), pages 7909-7913. IEEE.

Yun Tang, Juan Pino, Changhan Wang, Xutai Ma, and Dmitriy Genzel. 2021. A general multi-task learning framework to leverage text data for speech to text tasks. In ICASSP 2021-2021 IEEE International Conference on Acoustics, Speech and Signal Processing (ICASSP), pages 6209-6213. IEEE.

Nienke van Atteveldt, Elia Formisano, Rainer Goebel, and Leo Blomert. 2004. Integration of letters and speech sounds in the human brain. Neuron, 43(2):271-282.

Ashish Vaswani, Noam Shazeer, Niki Parmar, Jakob Uszkoreit, Llion Jones, Aidan N Gomez, Łukasz Kaiser, and Illia Polosukhin. 2017. Attention is all you need. In NIPS, pages 5998-6008.

Juan Raul Vazquez Carrillo, Alessandro Raganato, Jörg Tiedemann, Mathias Creutz, et al. 2019. Multilingual $\mathrm{nmt}$ with a language-independent attention bridge. In The 4th Workshop on Representation Learning for NLP (RepL4NLP-2019) Proceedings of the Workshop. The Association for Computational Linguistics.

Laura Cross Vila, Carlos Escolano, José AR Fonollosa, and Marta R Costa-jussà. 2018. End-to-end speech translation with the transformer. In IberSPEECH, pages 60-63.

Hari Krishna Vydana, Martin Karafiát, Katerina Zmolikova, Lukáš Burget, and Honza Černockỳ. 2021. Jointly trained transformers models for spoken language translation. In ICASSP 2021-2021 IEEE International Conference on Acoustics, Speech and Signal Processing (ICASSP), pages 7513-7517. IEEE.

Chengyi Wang, Yu Wu, Shujie Liu, Zhenglu Yang, and Ming Zhou. 2020a. Bridging the gap between pretraining and fine-tuning for end-to-end speech translation. In Proceedings of the AAAI Conference on Artificial Intelligence, volume 34, pages 9161-9168.

Chengyi Wang, Yu Wu, Shujie Liu, Ming Zhou, and Zhenglu Yang. 2020b. Curriculum pre-training for end-to-end speech translation. arXiv preprint arXiv:2004.10093.

Ron J Weiss, Jan Chorowski, Navdeep Jaitly, Yonghui $\mathrm{Wu}$, and Zhifeng Chen. 2017. Sequence-tosequence models can directly translate foreign speech. arXiv preprint arXiv:1703.08581.

Yinfei Yang, Gustavo Hernández Abrego, Steve Yuan, Mandy Guo, Qinlan Shen, Daniel Cer, Yun-Hsuan Sung, Brian Strope, and Ray Kurzweil. 2019. Improving multilingual sentence embedding using bidirectional dual encoder with additive margin softmax. arXiv preprint arXiv:1902.08564.
Katherine Yu, Haoran Li, and Barlas Oguz. 2018. Multilingual seq2seq training with similarity loss for cross-lingual document classification. In Proceedings of The Third Workshop on Representation Learning for NLP, pages 175-179.

Biao Zhang, Ivan Titov, Barry Haddow, and Rico Sennrich. 2020a. Adaptive feature selection for end-to-end speech translation. arXiv preprint arXiv:2010.08518.

Biao Zhang, Philip Williams, Ivan Titov, and Rico Sennrich. 2020b. Improving massively multilingual neural machine translation and zero-shot translation. arXiv preprint arXiv:2004.11867.

Pei Zhang, Boxing Chen, Niyu Ge, and Kai Fan. 2019. Lattice transformer for speech translation. arXiv preprint arXiv:1906.05551.

Changfeng Zhu, Heng Yu, Shanbo Cheng, and Weihua Luo. 2020. Language-aware interlingua for multilingual neural machine translation. In Proceedings of the 58th Annual Meeting of the Association for Computational Linguistics, pages 1650-1655. 